\title{
Nuclear Magnetic Resonance Study of Thermodynamic Interaction between Cellulose Acetate and Solvent
}

\author{
Kenji Kamide, Kunihiko OKAJIMA, and Masatoshi SAITO \\ Textile Research Laboratory, Asahi Chemical Industry Co. Ltd., \\ 11-7 Hacchonawate-machi, Takatsuki, Osaka 569, Japan.
}

(Received June 4, 1980)

\begin{abstract}
The thermodynamic interaction between cellulose acetate (CA) and solvent was studied by ${ }^{1} \mathrm{H}$ nuclear magnetic resonance (NMR) and infrared spectroscopy. Three CA whole polymers (degrees of substitution, $D S=0.49,2.46$, and 2.92) and their fractions were used. Chemical shifts of the methyl proton in the $O$-acetyl and of the hydroxyl proton in the CA molecule in various solvents were measured as functions of dielectric constant $\varepsilon$, electronegativity $\Delta v$ of the solvent, the degree of substitution $D S$ and the weight-average molecular weight $M_{w}$ of CA. These chemical shifts were constant in the range $M_{w} \gtrsim 1 \times 10^{5}$. The chemical shifts of methyl proton of $O$-acetyl and hydroxyl groups are a unique function of $\varepsilon$ and $\Delta v$. The CA molecule has a stronger interaction with high polar solvents, and there are intimate correlations between the short-range interaction parameter $A$ and these chemical shifts. The solvent dependence of $A$, observed for cellulose acetates, can be interpreted by the $O$-acetyl ' solvent and hydroxyl ' solvent interactions.

KEY WORDS Thermodynamic Interaction / Cellulose Acetate $/{ }^{1} \mathrm{H}$ Nuclear Magnetic Resonance / Dielectric Constant / Electronegativity /
\end{abstract}

Recently Kamide and his coworkers ${ }^{1-4}$ have studied in a systematic manner the dilute solution properties of cellulose acetate (CA) (degree of substitution, $D S=0.49,2.46$ and 2.92 ) and found that the unperturbed chain dimensions $A$, defined as the radius of gyration of a polymeric chain at the unperturbed state $\left\langle S^{2}\right\rangle_{0}^{1 / 2}$ devided by the square root of the molecular weight $M$, estimated from the solution data, depends strongly on the solvent nature, especially polarity. This phenomenon is very noticeable in polar polymers. The unusually large solvent dependence of the unperturbed chain dimensions has already been reported for cellulose, amylose, and their derivatives by Kamide et al., ${ }^{5-9}$ who analyzed literature data available. On the other hand, the existence of a hydrogen bond between the $O$-acetyl group in the CA molecule and halogenated hydrocarbons $\mathrm{s}^{10,11}$ and the interaction between the $O$-acetyl group and aniline or acidic solvents has been proposed ${ }^{12}$ on the basis of infrared spectroscopic observations. These results indicate that there exists specific interaction other than the van der Waals force between and solvent molecules, influencing the unperturbed chain dimensions. Up to now, ${ }^{1} \mathrm{H}$ NMR spectroscopy has been applied to the CA molecule for (1) determination of its configuration, ${ }^{13}(2)$ identification of the chemical shifts of ring proton, (3) evaluation of the average combined acetic acid content ${ }^{14}$ and (4) determination of the distribution of acetyl group over $\mathrm{C}_{2}$, $\mathrm{C}_{3}$, and $\mathrm{C}_{6}$ positions. ${ }^{15,16}$ Nuclear magnetic resonance (NMR) spectroscopy is also expected to provide a powerful tool for studying the interaction of functional groups in cellulose derivatives and solvents.

In this paper an attempt is made to study the thermodynamic interaction between the CA molecule and the solvent molecule by ${ }^{1} \mathrm{H}$ NMR spectroscopy.

\section{EXPERIMENTAL}

\section{Polymer Sample and Solvent}

Water-soluble incompletely-substituted cellulose acetate (CA $(D S=0.49))$, cellulose diacetate (CA $(D S=2.46))$ and triacetate (CA $(D S=2.92))$ whole polymers and their fractions, prepared in the 
Table I. Molecular characteristics of cellulose acetate samples

\begin{tabular}{|c|c|c|c|c|}
\hline Polymer & $\begin{array}{c}\text { Sample Code } \\
\text { (whole polymer } \\
\text { of fraction) }\end{array}$ & $\begin{array}{c}\text { Degree of } \\
\text { substitution, } \\
D S\end{array}$ & $\begin{array}{c}\text { Weight-average } \\
\text { molecular weight, } M_{w} \times 10^{-5}\end{array}$ & $M_{w} / M_{n}$ \\
\hline $\begin{array}{l}\text { Water-soluble } \\
\text { cellulose acetate } \\
\quad(D S=0.49)\end{array}$ & CMA $9(w)$ & 0.49 & 0.80 & 1.3 \\
\hline \multirow{5}{*}{$\begin{array}{l}\text { Cellulose } \\
\text { diacetate } \\
\qquad(D S=2.46)\end{array}$} & EF3-W (w) & 2.46 & 1.20 & 2.72 \\
\hline & EF3-3 (f) & 2.46 & 0.38 & 1.3 \\
\hline & EF3-7 (f) & 2.46 & 0.70 & 1.3 \\
\hline & EF3-10 (f) & 2.46 & 1.09 & 1.3 \\
\hline & EF3-14 (f) & 2.46 & 1.75 & 1.3 \\
\hline \multirow{4}{*}{$\begin{array}{l}\text { Cellulose } \\
\text { triacetate } \\
\quad(D S=2.92)\end{array}$} & TA2-W (w) & 2.92 & 2.35 & 4.02 \\
\hline & TA2-5 (f) & 2.92 & 0.822 & 1.4 \\
\hline & TA2-7 (f) & 2.92 & 2.62 & 1.4 \\
\hline & TA2-10 (f) & 2.92 & 5.00 & 1.4 \\
\hline
\end{tabular}

previous papers, ${ }^{1,3,4}$ were used. The molecular characteristics of these whole polymers and their fractions are presented in Table I.

Samples were dissolved in appropriate solvents: trichloromethane (TCM) for CA $(D S=2.92)$, acetone for CA $(D S=2.46)$ and water for CA $(D S=0.49)$. The solutions were cast on glass plate and the films thus prepared were subjected to infrared analysis.

As deuterated solvents, dimethylacetamide (DMAc)- $d_{9}$, pyridine- $d_{5}$, trifluoroacetic acid (TFA)$d_{1}$, tetrahydrofuran (THF)- $d_{5}$, acetone- $d_{6}$, dimethylformamide (DMF) $-d_{7}$ and deuterium oxide $\left(\mathrm{D}_{2} \mathrm{O}\right)$ were used as received from the producer (E. Merck, Dalmstad, W. Germany). Other solvents employed were non-deuterated spectroscopy grade chemicals and were used after distillation.

\section{${ }^{1} H$ NMR Spectroscopy}

${ }^{1} \mathrm{H}$ NMR spectra of CA in a wide variety of solvents were recorded on a ${ }^{1} \mathrm{H}$ NMR spectrometer model PMX60 $(60 \mathrm{MHz}$, resolving poer $0.04 \mathrm{~Hz}$, JOEL, Japan) and the chemical shifts of the methyl protons of $O$-acetyl and hydroxyl protons relative to the internal tetramethylsilane (TMS) were determined. All spectra were obtained at a probe temperature of $37^{\circ} \mathrm{C}$. Preliminary measurements showed no variation in the methyl- and hydroxylproton magnetic resonance of $\mathrm{CA}$ at a concentration ranging from $3-10 \mathrm{vol} \%$. Hence, the chemical shifts were determined at a concentration of $5-7 \mathrm{vol} \%$. When the peak due to the methyl proton in the $O$-acetyl group split into more than two parts, the weight-average of the characteristic chemical shifts of the methyl proton was calculated model PMX60 $(60 \mathrm{MHz}$, resolving power $0.04 \mathrm{~Hz}$, from these split speaks. The hydroxyl-proton signal was detected through a comparison of the NMR spectra obtained in the absence and presence of deuterium oxide or trifluoroacetic acid. All chemical shifts in this study are expressed in parts per million down-field from the internal tetramethylsilane.

\section{Infrared Spectroscopy}

The infrared (IR) spectra of CA ( $D S=2.92,2.46$, and 0.49$)$ in the film form and CA $(D S=2.92$ and 2.46) in the solvents were obtained with a Shimadzu model 430 infrared spectrophotometer. A $1-5$ vol $\%$ polymer solution was introduced by the capillary method between two rock salts, and the solvent compensation method was used for making measurements.

\section{RESULTS AND DISCUSSION}

Figures 1a-lc show the ${ }^{1} \mathrm{H}$ NMR spectra of CA $(D S=2.92)$, CA $(D S=2.46)$, and CA $(D S=0.49)$ in various solvents together with their assignments.

The chemical analysis of CA $(D S=2.92)$ indicated that there remained one hydroxyl group per ten 
NMR Study of Thermodynamic Interaction between CA and Solvent
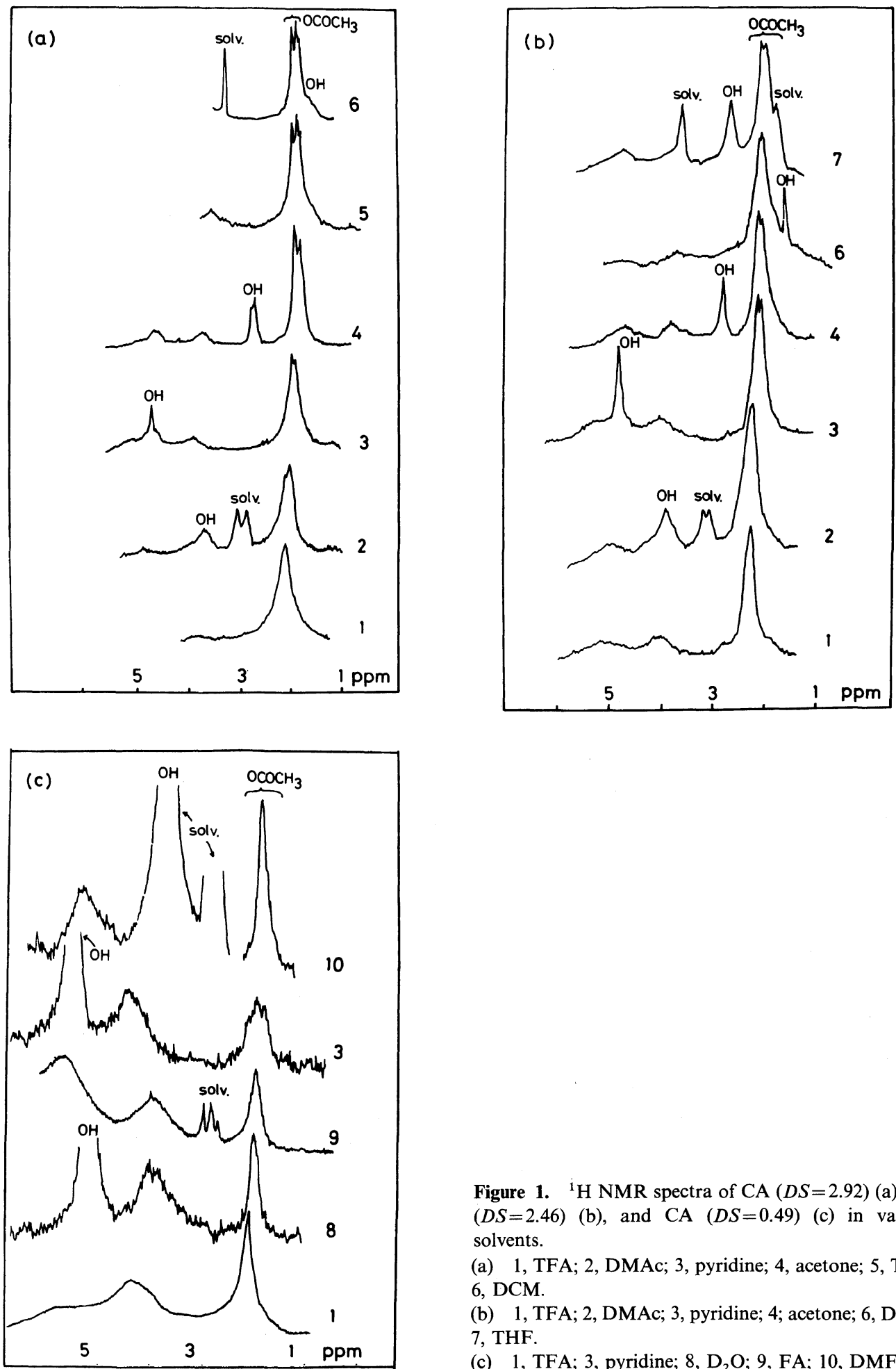

Figure 1. ${ }^{\mathrm{i}} \mathrm{H}$ NMR spectra of CA $(D S=2.92)(\mathrm{a}), \mathrm{CA}$ $(D S=2.46)(\mathrm{b})$, and CA $(D S=0.49)$ (c) in various solvents.

(a) 1, TFA; 2, DMAc; 3, pyridine; 4, acetone; 5, TCE; 6, DCM.

(b) 1, TFA; 2, DMAc; 3, pyridine; 4; acetone; 6, DCM; 7, THF.

(c) 1 , TFA; 3 , pyridine; $8, \mathrm{D}_{2} \mathrm{O} ; 9$, FA; 10 , DMF. 
Table II. NMR peaks of $O$-acetyl and hydroxyl protons of CA $(D S=2.92$,

2.46 , and 0.49 ) in various solvents

\begin{tabular}{|c|c|c|c|c|}
\hline Solvent & $\begin{array}{l}\text { Proton } \\
\text { species }\end{array}$ & $\mathrm{CA}(D S=2.92)$ & $\begin{array}{c}\text { NMR peaks/ppm } \\
\text { CA }(D S=2.46)\end{array}$ & $\mathrm{CA}(D S=0.49)$ \\
\hline Formamide & $\begin{array}{l}\text { (A) } O \text {-acetyl } \\
\text { (B) Hydroxyl }\end{array}$ & - & - & $\begin{array}{l}2.17 \\
3.72\end{array}$ \\
\hline $\mathrm{D}_{2} \mathrm{O}$ & $\begin{array}{l}\text { (A) } O \text {-acetyl } \\
\text { (B) Hydroxyl }\end{array}$ & - & - & $\begin{array}{l}2.19 \\
4.70\end{array}$ \\
\hline TFA & $\begin{array}{l}\text { (A) } O \text {-acetyl } \\
\text { (B) Hydroxyl }\end{array}$ & $\begin{array}{c}2.23 \\
-\end{array}$ & $\begin{array}{c}2.24 \\
-\end{array}$ & $\begin{array}{c}2.22 \\
-\end{array}$ \\
\hline DMAc & $\begin{array}{l}\text { (A) } O \text {-acetyl } \\
\text { (B) Hydroxyl }\end{array}$ & $\begin{array}{c}2.23,2.13 \\
3.84\end{array}$ & $\begin{array}{l}2.23 \\
3.90\end{array}$ & - \\
\hline Acetone & $\begin{array}{l}\text { (A) } O \text {-acetyl } \\
\text { (B) Hydroxyl }\end{array}$ & $\begin{array}{c}2.06,1.97 \\
2.75\end{array}$ & $\begin{array}{c}2.07,1.97 \\
2.85\end{array}$ & - \\
\hline THF & $\begin{array}{l}\text { (A) } O \text {-acetyl } \\
\text { (B) Hydroxyl }\end{array}$ & - & $\begin{array}{c}2.03,1.94 \\
2.51\end{array}$ & - \\
\hline Pyridine & $\begin{array}{l}\text { (A) } O \text {-acetyl } \\
\text { (B) Hydroxyl }\end{array}$ & $\begin{array}{c}2.13,2.03 \\
4.80\end{array}$ & $\begin{array}{c}2.13,2.04 \\
4.83\end{array}$ & $\begin{array}{c}2.25,2.11,2.01 \\
4.92\end{array}$ \\
\hline DCM & $\begin{array}{l}\text { (A) } O \text {-acetyl- } \\
\text { (B) Hydroxyl }\end{array}$ & $\begin{array}{c}2.07,1.98,1.94 \\
1.64\end{array}$ & $\begin{array}{c}2.09,2.05,2.02 \\
1.62\end{array}$ & - \\
\hline TCE & $\begin{array}{l}\text { (A) } O \text {-acetyl } \\
\text { (B) Hydroxyl }\end{array}$ & $\begin{array}{c}2.10,2.00,1.94 \\
1.70\end{array}$ & - & - \\
\hline DMF & $\begin{array}{l}\text { (A) } O \text {-acetyl } \\
\text { (B) Hydroxyl }\end{array}$ & - & - & $\begin{array}{l}2.07 \\
3.50\end{array}$ \\
\hline
\end{tabular}

glucopyranoside residues. Three separate signals between 1.9 and $2.1 \mathrm{ppm}$ were detected individually for CA $(D S=2.92)$ in chlorinated hydrocarbons and assigned to the methyl-proton groups of $O$-acetyl substituents in three different positions $\mathrm{C}_{2}, \mathrm{C}_{3}$, and $\mathrm{C}_{6}$ in the glucopyranose units, to which the acetyl group is attached. For example, in dichloromethane (DCM) the three methyl proton peaks were $2.07,1.98$, and $1.94 \mathrm{ppm}$. These values are in reasonably good accordance with those reported by Goodlett et al. ${ }^{15}$ for fully-substituted CA in DCM. These authors assigned the signals at 2.09, 1.99, and $1.94 \mathrm{ppm}$ to the methyl groups of $O$-acetyl attached to the $\mathrm{C}_{6}, \mathrm{C}_{2}$, and $\mathrm{C}_{3}$ positions, respectively. In solvents other than halogenated hydrocarbons, the signals due to the methyl proton were often appreciably superposed with each other, giving one or two peaks. This implies the exsitence of a specific interaction between the $O$-acetyl groups in the CA molecule and the solvent molecule in question.

In CA $(D S=2.46)$, it is expected from chemical analysis that there is approximately one hydroxyl group per two glucopyranose units. Therefore, the possibility of seven types of acetylated glucopyranose units yields twelve non-equivalent methyl protons with different magnetic environment. The chemical shift of the methyl-proton signals of CA $(D S=2.46)$ lies in a relatively narrow range, giving the peaks a rather complicated nature. This is very marked in THF, DCM, and acetone, but the signals due to the methyl group attached to $O$-acetyl enveloped two peaks in pyridine and a single peak in both DMAc and in TFA. The value, $2.24 \mathrm{ppm}$ obtained for TFA agrees well with the literature data. ${ }^{17}$ It should be noted that the hydroxyl-proton signals of the CA molecule occassionally overlap and are inseparable from water or HOD in solvents.

Figures' $2 a-2 d$ and Figures $3 a-3 c$ show the chemical shifts of the methyl proton in the $O$-acetyl group and the hydroxyl protons of CA $(D S=2.92)$ and CA $(D S=2.46)$ in various solvents as a function of the weight-average molecular weight $M_{w}$ of CA. In these figures the circles are for CA $(D S=2.46)$ 
NMR Study of Thermodynamic Interaction between CA and Solvent

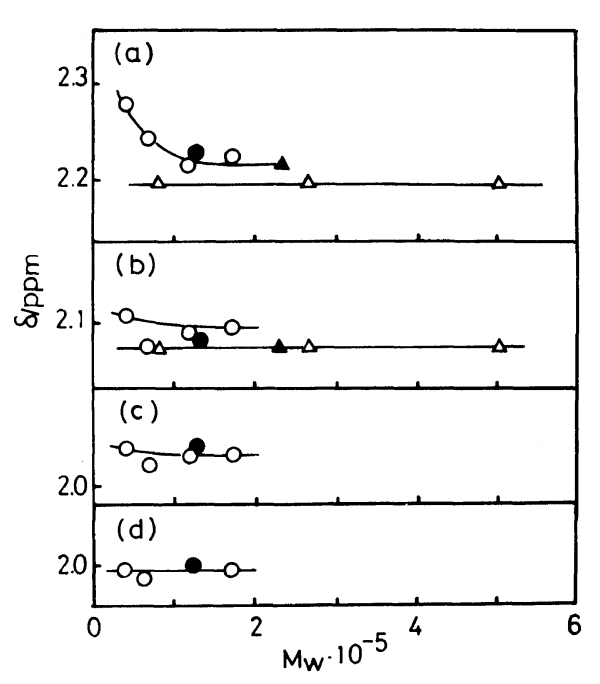

Figure 2. The molecular-weight dependence of the chemical shift of $O$-acetyl-proton of CA $(D S=2.92$ and $2.46)$ in TFA (a), pyridine (b), and of CA $(D S=2.46)$ in acetone (c) and THF (d): open mark, fractions; closed mark, whole polymer; triangle, CA $(D S=2.92)$; circle, CA $(D S=2.46)$.

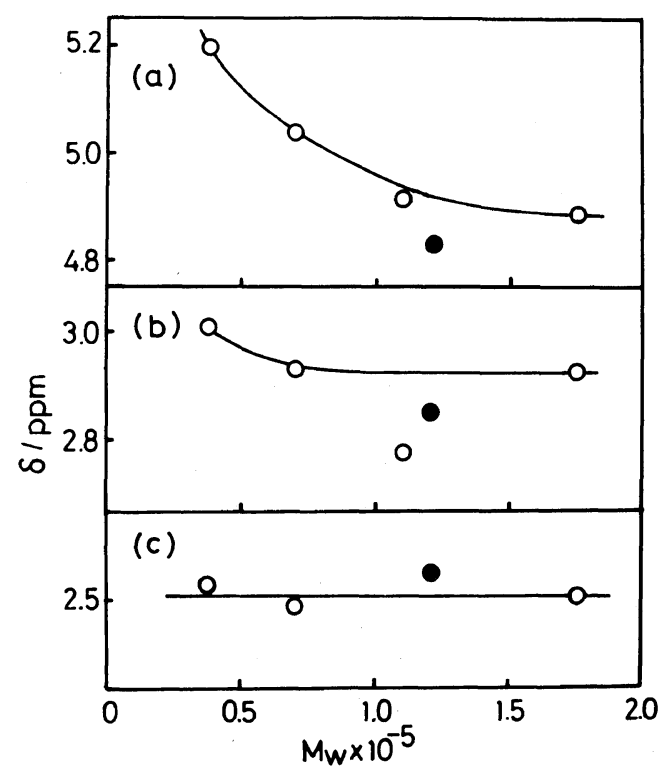

Figure 3. The molecular-weight dependence of the hydroxyl proton of CA $(D S=2.46)$ in pyridine (a), acetone (b), and THF (c). and the triangles are for CA $(D S=2.92)$, and the open and closed marks indicate the fractions and the whole polymers, respectively. In the CA $(D S=2.92)$-TFA and CA $(D S=2.92)$-pyridine systems, the chemical shift of the methyl proton shows no variation with the molecular weights. In contrast to this the methyl-proton signal of CA $(D S=2.46)$ in TFA shifts toward a lower magnetic field as $M_{w}$ decreases. The large disparity between CA $(D S=2.92)$ and CA $(D S=2.46)$ may originate from the difference in the molecular weight range studied, that is, the molecular weight range employed for CA $(D S=2.46)$ is lower than that for CA $(D S=2.92)$. The possibility of interaction between solvent and polymer might be higher when the molecular weight of the polymer is lower. The molecular-weight dependence of the methyl proton chemical shift for CA $(D S=2.46)$ in pyridine, acetone, and THF is far smaller than that in TFA. In the former two systems, the chemical shift of the hydroxyl proton shifts toward a lower field as $M_{w}$ decreases, as can be anticipated from the behavior of the methyl-proton chemical shift. In these systems, the decrease in $M_{w}$ strengthens the hydroxyl-solvent interaction. The molecular-weight dependence of these chemical shifts in two solvents becomes remarkable with increase in the electronegativity of the solvent. The hydroxyl-proton chemical shift depends more markedly on the molcular weigh than on the chemical shift of the $O$ acetyl proton for CA with the same $D S$ and $M_{w}$ dissolved in a given solvent. The polydispersity of the polymer samples has no significant effect on the chemical shifts of the $O$-acetyl and hydroxyl protons. The use of CA with $M_{w}$ approximately equal $1 \times 10^{5}$ allows us to neglect the molecularweight dependence of the chemical shifts. Hence, further experiments were done with the whole polymer samples.

The acetyl group in CA is known to be nucleophilic. Thus, the interaction between the $O$ acetyl group and the solvent is expected to depend on the properties of the solvent, such as dielectric constant and electronegativity. Figures $4 a$ and $4 b$ show the plot of the weight-average methyl-proton chemical shifts of CA against the dielectric constant, $\varepsilon(\mathrm{a})$ and the electronegativity, $\Delta v$ (b) of the solvent, respectively. In these figures, the squares stand for CA $(D S=0.49)$, the circles, for CA $(D S=2.46)$, and the triangles, for CA $(D S=2.92)$. The methyl- 

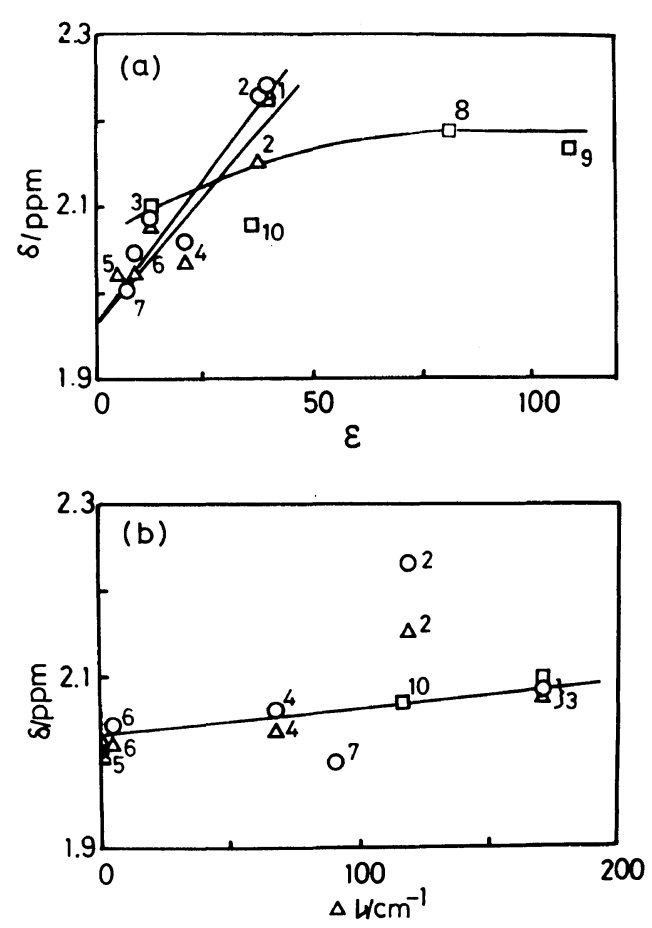

Figure 4. The plot of the weight-average methylproton chemical shifts of CA molecule against the dielectric constant, $\varepsilon$ (a) and the electro-negativity, $\Delta v$ (b) of the solvents: rectangle, CA $(D S=0.49)$; circle, CA $(D S=2.46)$; triangle, CA $(D S=2.92)$.

proton signals shift almost exclusively toward a lower magnetic field as the dielectric constant of the solvent becomes larger. The dependence of the chemical shift on the dielectric constant becomes stronger, depending on the $D S$ of the CA polymers and is highest for CA $(D S=2.46)$. For example, the methyl-proton signal shifts from 2.00 to $2.24 \mathrm{ppm}$ as the dielectric constant of solvent changes from 7.3 to 39.5. This suggests that solvents with high dielectric constants, such as TFA, stongly interact with the $O$ aetyl group of the $\mathrm{CA}$ molecule and that the interaction is strongest for CA $(D S=2.46)$ in the solvent with $\varepsilon \geqslant 20$. This tendency is particulary notable in DMAc as an aprotic reagent. The $O$ acetyl-proton chemical shift $\delta_{\mathrm{CH}_{3}}$ exhibits a linear correlation with the dielectric constant $\varepsilon$ of the solvent as follows.

$$
\begin{aligned}
\delta_{\mathrm{CH}_{3}}=1.97_{2}+5.68 \times 10^{-3} \varepsilon \\
D S=2.92,7.7 \leqq \varepsilon \leqq 39.5
\end{aligned}
$$

$$
\begin{gathered}
\delta_{\mathrm{CH}_{3}}=1.97_{2}+6.60 \times 10^{-3} \varepsilon \\
D S=2.46,7.3 \leqq \varepsilon \leqq 39.5 \\
\delta_{\mathrm{CH}_{3}=} 2.11_{2}+7.07 \times 10^{-4} \varepsilon \\
D S=0.49,12.3 \leqq \varepsilon \leqq 110 \\
=2.06+2.93 \times 10^{-3} \varepsilon-1.78 \times 10^{-5} \varepsilon^{2}
\end{gathered}
$$

Equations $1-4$ were established by the least-square method. The correlation coefficients, $\gamma$ for these relations are $0.927,0.956,0.457$, and 0.964 , respectively. By using eq 1,2 , and 4 , we can estimate the expected downfield chemical shift of the methyl proton from $\varepsilon$ of the solvent.

The methyl-proton signal linearly shifts from 2.00 to $2.10 \mathrm{ppm}$ as the electronegativity of solvent increases from 0 to $170 \mathrm{~cm}^{-1}$, except for all CA in DMAc $\left(\Delta v=138 \mathrm{~cm}^{-1}\right)$ and THF $\left(\Delta v=92 \mathrm{~cm}^{-1}\right)(2$ and 7 in the figure). The $O$-acetyl-proton chemical shift $\delta_{\mathrm{CH}_{3}}$ is expressed, except in DMAc and THF, as a function of the electronegativity $\Delta v$ in the form:

$$
\begin{gathered}
\delta_{\mathrm{CH}_{3}}=2.02+3.43 \times 10^{-4} \Delta v \\
0.49 \leqq D S \leqq 2.92, \quad 0 \leqq \Delta v \leqq 170
\end{gathered}
$$

The correlation coefficient $\gamma$ for eq 5 is 0.872 . A better correlation of $\delta_{\mathrm{CH}_{3}}$ is found with the dielectric constant (eq 1, 2, and 4), rather than with the electronegativity (eq 5).

Figure 5 illustrates the change in the limiting viscosity number $[\eta]$ of CA with $M_{w}=1.08 \times 10^{5}$ in the solvent having $\varepsilon=40$ at $25^{\circ} \mathrm{C}$ and the unperturbed chain dimensions, $A$ in the same solvent with $D S$, both interpolated from the experimental results. In the figure, the $O$-acetyl-proton chemical shift $\delta_{\mathrm{CH}_{3}}$ of CA in a solvent with $\varepsilon=40$, calculated from eq $1-3$, is also shown for comparison. There is a good correlation among $[\eta], A$, and the chemical shift of the $O$-acetyl-proton, suggesting that the former two parameters are closely correlated with the latter. It should be noted that the expansion factor, expressing the long-range interaction parameter, is almost constant, very near unity, irrespective of $D S$.

In Figures $6 \mathrm{a}$ and $6 \mathrm{~b}$ are shown the relations between the chemical shift of the hydroxyl proton in $\mathrm{CA}$ and the dielectric constant (a) or the electronegativity (b) of various solvents. In the figure, 


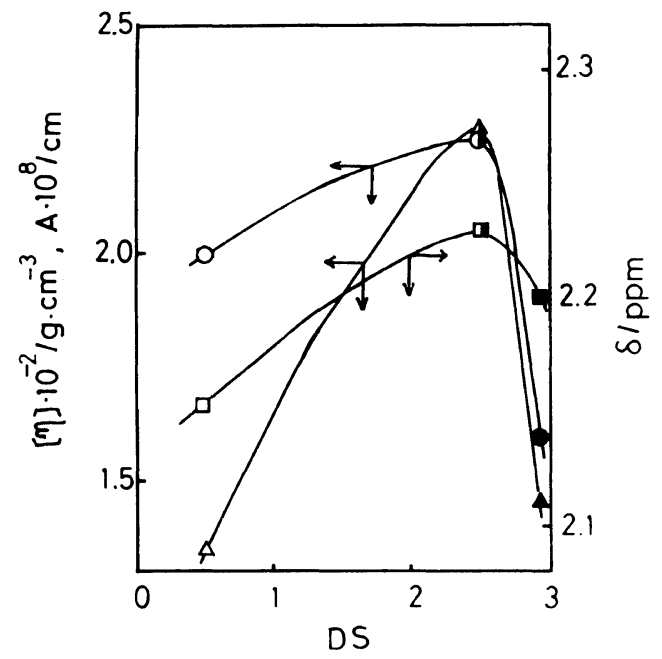

Figure 5. The effect of $D S$ on the limiting viscosity number [ $\eta$ ] of CA polymer with $M_{w}=1.08 \times 10^{5}$ in the solvent having $\varepsilon=40$ at $25^{\circ} \dot{\mathrm{C}}$ the unpertubed chain dimensions, $A$ in the same solvent and the $O$-acetylproton chemical shift, $\delta_{\mathrm{CH}_{3}}$ of CA polymer dissolved in a solvent having $\varepsilon=40$ : circle, $[\eta]$; triangle, $A$; rectangle, $\delta_{\mathrm{CH}_{3}}$.

the rectangles stand for CA $(D S=0.49)$, the circles, for CA $(D S=2.46)$, and the triangle, for CA $(D S=2.92)$. Evidently, the chemical shift of the hydroxyl proton is a unique function of the dielectric constant or the electronegativity irrespective of $D S$. The chemical shift increases with an in crease in the dielectric constant or the electronegativity of the solvent over the entire range of $\varepsilon$ and $\Delta v$ investigated. The chemical shift of the hydroxyl proton was found to be $4.8 \mathrm{ppm}$ in pyridine $(\Delta v=170)$ and $1.6-2.0 \mathrm{ppm}$ in halogenated hydrocarbons $(\Delta v=0)$, irrespective of the $D S$ of CA. The electronegativity of the solvent is an index for forming a hydrogen bond against the hydroxyl group in various alcohols. ${ }^{19}$ In this sence, the hydroxyl group in CA has a hydrogen bond forming capacity similar to low-molecular-weight alcohols. The following relations were experimentally obtained by the least-square method, between the hydroxyl-proton chemical shift, $\delta_{\mathrm{OH}}$ and the dielectric constant, $\varepsilon$ or the electronegativity, $\Delta v$ of the solvent.

$$
\begin{aligned}
\delta_{\mathrm{OH}}= & 4.04-17.1 / \varepsilon+1.68 \times 10^{-5} \varepsilon^{2} \\
& 0.49 \leqq D S \leqq 2.92, \quad 7.3 \leqq \varepsilon \leqq 110
\end{aligned}
$$
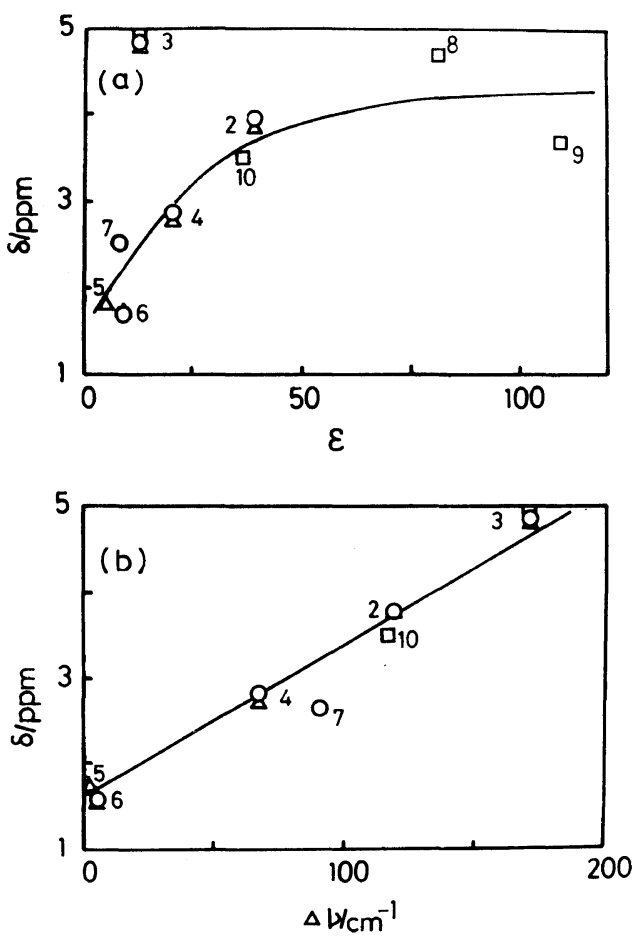

Figure 6. The plot of the weight-average hydroxylproton chemical shifts of CA molecule against the dielectric constant, $\varepsilon$ (a) and the electro-negativity, $\Delta v$ (b) of the solvents: rectangle, CA $(D S=0.49)$; circle, CA $(D S=2.46)$; triangle, CA $(D S=2.92)$.

and

$$
\begin{aligned}
\delta_{\mathrm{OH}}= & 1.61+1.81 \times 10^{-2} \Delta v \\
& 0.49 \leqq D S \leqq 2.92, \quad 0 \leqq \Delta v \leqq 170
\end{aligned}
$$

The correlation coefficients, $\gamma$ of these equations are 0.990 and 0.980 , respectively. For CA $(D S=2.92)$ and CA $(D S=2.46), \delta_{\mathrm{OH}}$ relates linearly to $\varepsilon$ as follows.

$$
\begin{aligned}
\delta_{\mathrm{OH}} & =1.12+7.32 \times 10^{-2} \varepsilon, \\
D S & =2.92,7.7 \leqq \varepsilon \leqq 39.5 \\
\gamma & =0.997,
\end{aligned}
$$

and

$$
\begin{aligned}
\delta_{\mathrm{OH}} & =1.61+6.02 \times 10^{-2} \varepsilon, \\
D S & =2.46, \quad 7.3 \leqq \varepsilon \leqq 39.5 \\
\gamma & =0.918,
\end{aligned}
$$

Moore and Russel ${ }^{18}$ explained the viscosity 
behavior of CA in various solvents on the basis of acidity and basicity of the solvents. But, as is evident from Figure 4, the interaction between CA and the solvent is not so simple as to accept Moore and Russel's explanation unconditionally. In fact, very recently we demonstrated that the limiting viscosity number $[\eta]$ of CA, with a given $D S$ and a given $M_{w}$, is a unique function of the dielectric constant of the solvent. $^{4}$

Figures 4 and 6 reveal that DMAc and TFA, both having large $\varepsilon$ and large $\Delta v$, engage in strong interactions with the $O$-acetyl and hydroxyl groups in CA.

In order to obtain more detailed information on the interaction between CA molecule and solvents, the IR spectra of the carbonyl groups in the films of CA $(D S=2.92)$ and CA $(D S=2.46)$ and CA $(D S=2.92)$ in solvents were measured. The results are shown in Figure 7. In most cases the maximum absorption band of the carbonyl group was found at $1741 \mathrm{~cm}^{-1}$ and in the dissolved state especially in DMAc the maximum band shifts to a higher wavenumber region $\left(1750 \mathrm{~cm}^{-1}\right)$. But no shift was observed in DCM. In 1943, Clermont ${ }^{11}$ observed a significant shift of the band at $1760 \mathrm{~cm}^{-1}$ to 1740 $\mathrm{cm}^{-1}$ due to the presence of DCM as solvent. Clermont's experimental results have been for long years cited in the literature without being subjected

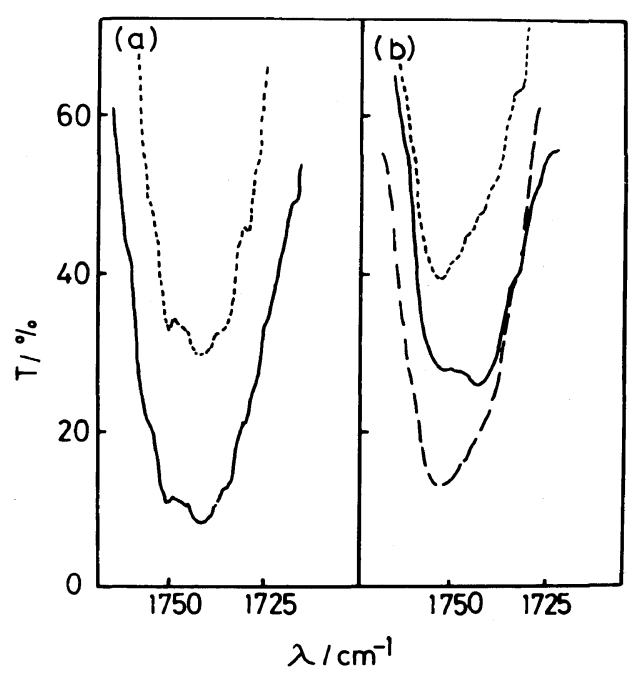

Figure 7. IR spectra of solid CA ( $D S=2.46$ and 2.92) (a) and that in solvents (b): (a), -----, CA $(D S=2.46)$; $\longrightarrow$, CA $(D S=2.92)$; (b),,- DCM; ---, DMAc; -----, epichlorohydrine. to detailed examination, but they are in sharp contradiction with the results in Figure 7. If Clermont's experimental results are correct as generally accepted, the $O$-acetyl groups in solid CA should exist in a free state, strongly interacting with DCM. But, contrary to this, the ring-conformational analysis of cellulose by Hermans ${ }^{21}$ and Robinson and Conmar $^{22}$ show definitely the possibility of the occurrence of an interaction between the $O$-acetyl groups in the solid state, even if the interacting force is weak. Inspection of Figure 7 leads to the conclusion that in the solid state, the interaction between the $O$-acetyl groups of CA $(D S=2.46)$ and also those of CA $(D S=2.92)$ is strong and the addition of a solvent weakens this interaction. Examination, based on $\mathrm{C}_{1}$ chain conformation by the molecular structure model, indicates that conformations in which the $O$-acetyl and hydroxyl groups belonging to the same cellobiose unit approach each other are possible for CA $(D S=$ 2.46 ), in which a hydroxyl group exists in each cellobiose unit. The above expectation is thoroughly consistent with the appearance of a band due to a carbonyl group at $1732 \mathrm{~cm}^{-1}$ found for CA $(D S=0.49)$. This value is significantly lower than $1741 \mathrm{~cm}^{-1}$ found for CA $(D S=2.46)$ and CA $(D S=2.92)$. In CA $(D S=0.49)$, an $O$-acetyl group exists in each of three glucopyranose units and the effect of the $O$-acetyl $\cdots O$-acetyl interaction on the solution behavior is negligible, compared with that of CA ( $D S=2.46$ and 2.92), as far as a cellobiose unit is concerned, but the $O$-acetyl $\cdots$ hydroxyl interaction may shift the band due to a carbonyl, to a lower wavenumber $\left(1732 \mathrm{~cm}^{-1}\right)$.

Figure 8 illustrates the possible conformation patterns of solid CA $(D S=2.92)$ and CA $(D S=2.46)$. For the former, the interaction between nearest neighboring $O$-acetyl groups of contiguous glucopyranose units is shown and for the latter, the possible interaction between the $O$-acetyl group, hydroxyl group, and ring-oxygene atoms of contiguous glucopyranose units.

Figures 9 and 10 show the possible molecular models of the interactions of CA $(D S=2.46)$ and CA $(D S=2.92)$ in various solvents. The models in these figures were constructed by taking into consideration the conclusions deducted from NMR spectra; i.e., the interaction of the solvent molecule with the $O$-acetyl group in the CA molecule decreases in the following order: TFA $>$ DMAc $>$ 


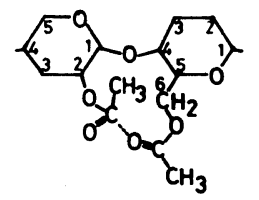

(a)
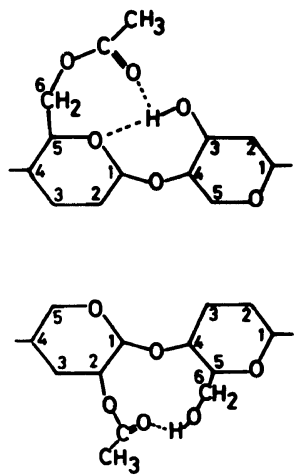

(b)

Figure 8. Possible conformation patterns of solid CA $(D S=2.92)$ (a) and CA $(D S=2.46)(\mathrm{b})$.
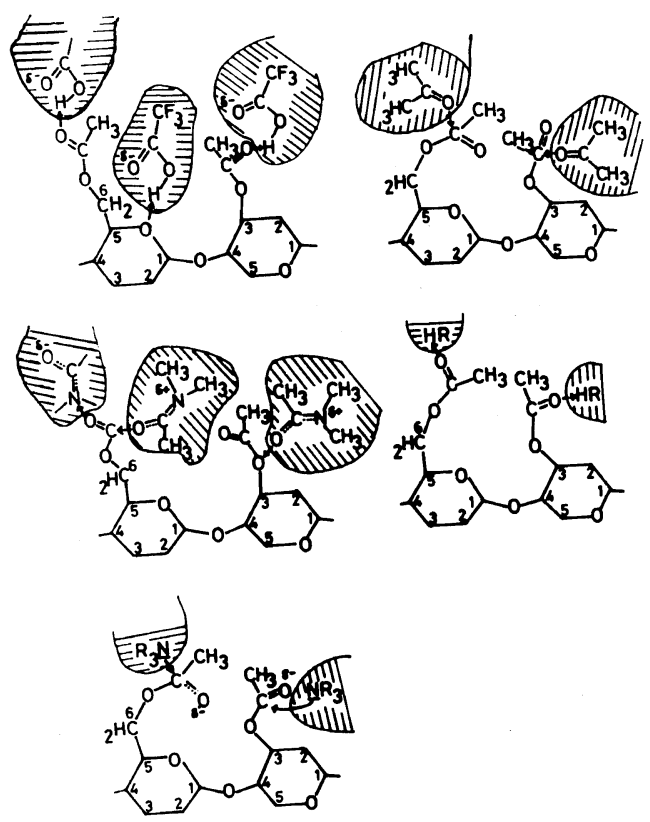

Figure 9. Possible molecular model of interaction of CA $(D S=2.92)$ with various solvent employed in this study.
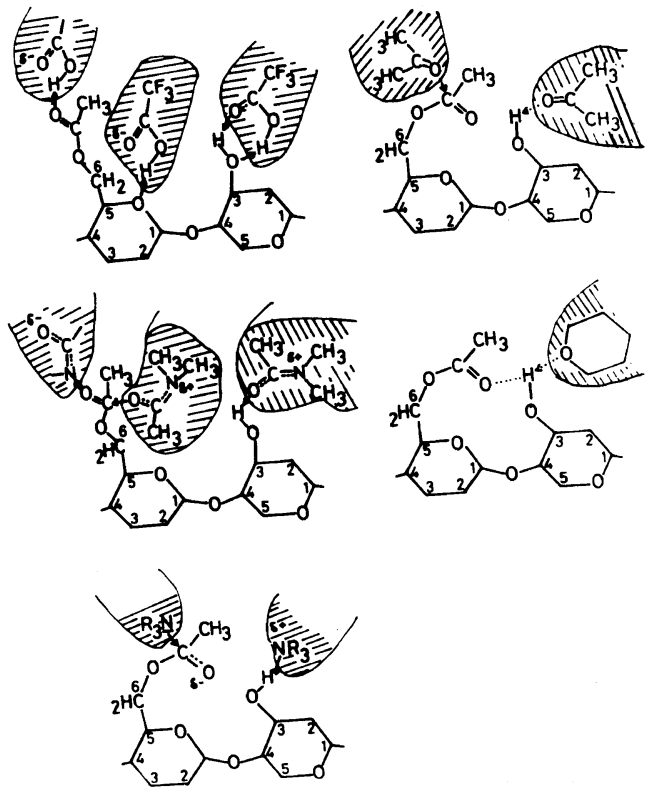

Figure 10. Possible molecular model of interaction of CA $(D S=2.46)$ with various solvent employed in this study.

pyridine $>$ acetone $>$ halogenated hydrocarbons $>$ THF. The interaction with hydroxyl group is in the order: TFA $>$ pyridine $>$ DMAc $>$ acetone $>$ THF $>$ halogenated hydrocarbons. The interaction of the hydroxyl group with TFA will be discussed in further detail later.

Figures $11 \mathrm{a}$ and $11 \mathrm{~b}$ show the effect of $D S$ on the $O$-acetyl- and hydroxy-proton chemical shifts of CA in TFA $(\varepsilon=39.5)$, DMAc $(\varepsilon=38.6)$, and pyridine $(\varepsilon=12.3)$. The data points of CA $(D S=0.49)$ in DMAc were interpolated from those of CA with larger $D S$. The spectrum of the hydroxyl proton of CA in TFA was overlapped considerably and could not be separated from that of the carboxyl proton. In Figure 11c, the difference, $\Delta \delta$ between the chemical shift of the caryboxyl proton of TFA and that of pure TFA is taken as representing the extent of the interaction of the carboxyl group in TFA and the hydroxyl group in CA. The chemical shift of the $O$-acetyl-proton reveals a maximum at $D S=2.46$ in TFA and DMAc, but is almost independent of $D S$ in pyridine. These results are readily expected from Figure 4. The absolute magnitude of the shift is larger in TFA than in pyridine and that in DMAc lies intermediate between these. The $D S$ dependence 


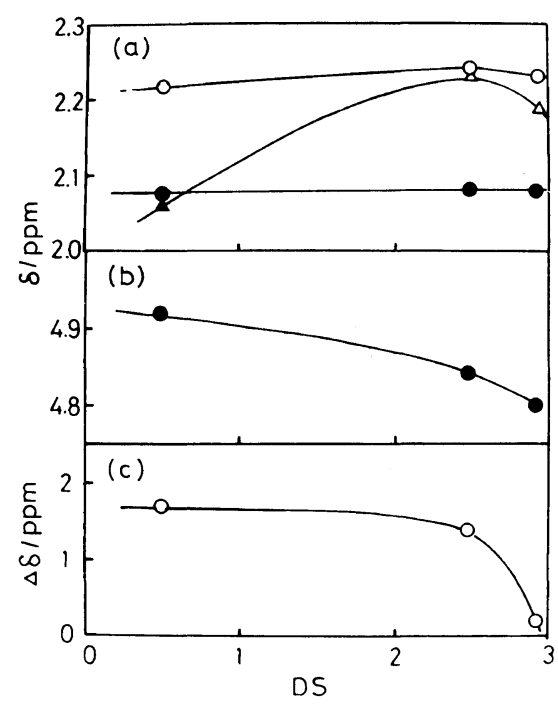

Figure 11. (a): The effect of the degree of substitution, $D S$ on the $O$-acetyl-proton chemical shift of $\mathrm{CA}$ in various solvents: open circle, TFA; closed circle, pyridine; open triangle, DMAc. Closed triangle is the interpolated value.

(b) : The effect of the degree of substitution, $D S$ on the hydroxyl-proton chemical shifts of CA in pyridine.

(c): The effect of the degree of substitution, DS on the difference of the chemical shift of the carboxyl proton of TFA of CA solution and that of pure TFA.

of the $O$-acetyl-proton chemical shift is much smaller in TFA than in DMAc. The above large difference in $D S$ dependence may be attributed to the differences in the solvent. TFA is a highly protondonating solvent and pyridine is a typical basic reagent. Other solvents, including DMAc, employed in this work are almost neutral or amphoteric.

The intraction of the hydroxyl group with pyridine and TFA decreases gradually with an increase in $D S$, indicating that the above interaction becomes weak with increasing $D S$. From this, we can conclude that the interaction of $O$-acetyl group in CA with the solvent plays an impotant role in the dissolution process of CA $(D S=2.92)$ in solvent and that in addition to this kind of interaction, the interaction between the hydroxyl group with solvent becomes large as $D S$ decreases.

In previous papers, ${ }^{1,3}$ we demonstrated that the limiting viscosity numbers $[\eta]$ of CA $(D S=2.92)$ fractions in DMAc are larger than those in halogenated hydrocarbons and that these $[\eta]$ values
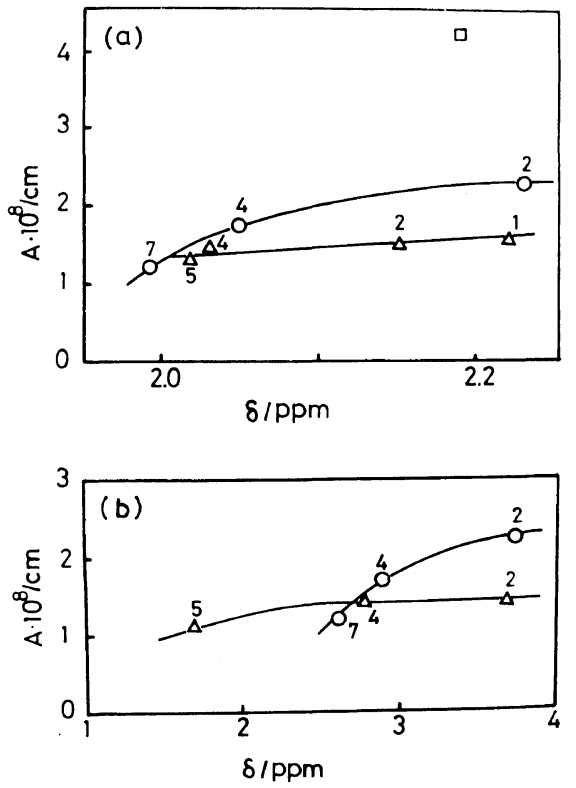

Figure 12. The plot of the unperturbed chain dimensions, $A$ as a function of the chemical shift of $O$ acetylmethyl (a) and hydroxyl group (b).

for acetone lie between those of these two solvents: DMAc $>$ acetone $>$ halogenated hydrocarbon. The solvent dependence of the perturbed and unperturbed radii of gyration, $\left\langle S^{2}\right\rangle_{z}^{1 / 2}$ and $\left\langle S^{2}\right\rangle_{0, z}^{1 / 2}$, parallel that of $[\eta]$. Contrary to this, the $\chi$-parameter and the expansion factor $\alpha_{\mathrm{s}}$ are almost independent of the solvent employed. In short, the short-range interaction is remarkably influenced by the solvent nature. This may be explained in terms of the $O$ acetyl (or hydroxyl) * solvent interaction.

Figure 12 shows a plot of the unperturbed-chain dimensions $\mathrm{A}$ as a function of the chemical shifts of the $O$-acetyl group (a) and the hydroxyl group (b). In this figure, the rectangles stand for CA $(D S=0.49)$, the circles for CA $(D S=2.46)$ and the triangles, for CA $(D S=2.92)$. The $A$ values were determined in previous papers. ${ }^{1,3}$ For CA $(D S=2.46)$, the unperturbed chain dimension becomes large as the signals of methyl and hydroxyl groups shifts to a lower magnetic field. Such a tendency though significant is much less remarkable for CA $(D S=2.92)$ than for CA $(D S=2.46)$. The strong intermolecular interaction between hydroxyl and acetyl groups formed in solid cellulose diacetate (see, Figure 7) is partially or fully destroyed by the 
interaction between the hydroxyl group and solvent and that between the acetyl group and solvent. The degree of destruction depends on the solvent nature, especially its polarity. On the other hand, the intermolecular or intramolecular interactions in CA $(D S=2.92)$ solid are weak enough to be fully broken by the addition of a solvent if it can dissolve the CA $(D S=2.92)$ polymer. The above mechanism, although rather speculative, can explain qualitatively the noticeable differences in the $A$ value when there is a chemical shift of the methyl and hydroxyl protons. For CA $(D S=2.46)$ in DMAc, the $O$-acetyl $\cdot$ solvent and hydroxyl $\cdot$ solvent interactions are stronger than those in acetone, and in addition, the high polarity of DMAc, through interaction with CA $(D S=2.46)$, induces the mutual repulsion of the polymer chains, resulting in a large radius of gyration $\left\langle S^{2}\right\rangle^{1 / 2}$ and a large $[\eta]$, compared with the CA $(D S=2.46)$-acetone system.

Hence, we can interpret the solvation of CA in polar solvents as involving the $O$-acetyl or hydroxyl - solvent bond formation with numerous arrangements (see Figure 9) and these doubtlessly strong interactions may give rise to the solvation of CA. It seems plausible to assume that the CA molecule along with the solvated solvent dissolve into non-solvated solvent molecules, and behave as a single solute molecule. Consequently, the solvated solvent $\cdot$ non-solvated solvent contact may predominate over the CA molecule'-non-solvated solvent contact. This hypothesis can explain the well-known experimental facts such as small second-virial coefficients, small expansion coefficients $\left(\alpha_{s} \simeq 1\right)$, and large unpertubed chain dimensions of cellulose derivatives in dilute solutions. The last fact may be due to the fact that the solvated solvent molecules interfere with the contraction of the space occupied by the CA molecule in solution. It is noteworthy that the intensity of the interaction can be estimated by the chemical shift in NMR spectra, but the number of moles of the solvent involved in the solvation of one mole of a substituted glucopyranose unit can not be counted. Therefore, unless an accurate knowledge of the degree of solvation is available, we cannot discuss the solvent dependence of $A$ value at the molecular level.

\section{REFERENCES}

1. K. Kamide, T. Terakawa, and Y. Miyazaki, Polym. J., 11, 285 (1979).

2. S. Ishida, H. Komatsu, T. Terakawa, Y. Miyazaki, and K. Kamide, Mem. Fac. Eng., Kanazawa Univ., 12, 103 (1979).

3. K. Kamide, Y. Miyazaki, and T. Abe, Polym. J., 11, 523 (1979).

4. K. Kamide, M. Saito, and T. Abe, to be submitted to Polym. J.,

5. K. Kamide and Y. Miyazaki, Polym. J., 10, 409 (1978).

6. K. Kamide and T. Terakawa, Polym. J., 10, 559 (1978).

7. K. Kamide and Y. Miyazaki, Polym. J., 10, 539 (1978).

8. H. Suzuki, K. Kamide, and Y. Miyazaki, Netsusokutei, 7, 37 (1980).

9. H. Suzuki, Y. Miyazaki, and K. Kamide, Eur. Polym. J., 16, 703 (1980).

10. H. M. Spurlin, "Cellulose and Cellulose Derivatives," E. Otto, Ed., Interscience Publishers, New York, N.Y., 1943, p 868.

11. P. Clermont, Ann. Chim., 12, 2420 (1943).

12. R. J. B. Marsden and A. R. Urquhart, J. Text. Inst., 33, T-105 (1942).

13. R. U. Lemiux and J. D. Stevens, Can. J. Chem., 43, 1059 (1965).

14. D. Gagnaire and M. Vincendon, Bull. Soc. Chim. Fr., 204 (1966).

15. V. W. Goodlett, J. T. Dougherty, and H. W. Patton, J. Polym. Sci., A-1, 9, 155 (1971).

16. N. Shiraishi, T. Katayama, and T. Yokota, Cellul. Chem. Technol., 12, 429 (1978).

17. "The Sadtler Guide to the NMR spectra of Polymers," W. W. Simons and M. Zanger, Ed., Sadtler Laboratory, New York, N.Y., 1973, p 171.

18. W. R. Moore and J. Russel, J. Colloid. Sci., Jpn., 9, 338 (1954).

19. See, for example, T. Kagiya, Bull. Chem. Soc. Jpn., 41, 767 (1969).

20. See, for example, R. H. Baker, and R. A. Pittman, "Cellulose and Cellulose Derivatives," N. M. Bikales and L. Segal, Ed., Wiley-Interscience, New York, N.Y., 1971, p 193.

21. P. H. Hermans, "Physics and Chemistry of Cellulose Fibers," Elsevier, New York, N.Y., 1949, p 13.

22. J. Robinson and E. Conmar, Discuss. Faraday Soc., 16, 125 (1954). 